\title{
A study of methods for estimating in the exponentiated Gumbel distribution
}

\author{
K. Fathi ${ }^{1 *}$, S.F. Bagheri², M. Alizadeh ${ }^{3}$, M. Alizadeh ${ }^{4}$ \\ Department of Statistics, North Branch, Islamic Azad University, Tehran, Iran ${ }^{1}$ \\ Department of Statistics, College of Basic Sciences, Yadegar-e-Imam Khomeini (RAH) Shahr-e-Rey Branch, \\ Islamic Azad University, Tehran, Iran ${ }^{2}$ \\ Branch of Mazandaran, Statistical Center of Iran $^{3}$ \\ Department of Mathematical since, University of Mazandaran, Mazandaran, Iran ${ }^{4}$
}

Received 18 August 2015

Accepted 24 October 2016

\begin{abstract}
The exponentiated Gumbel model has been shown to be useful in climate modeling including global warming problem, flood frequency analysis, offshore modeling, rainfall modeling and wind speed modeling. Here, we consider estimation of the PDF and the CDF of the exponentiated Gumbel distribution. The following estimators are considered: uniformly minimum variance unbiased (UMVU) estimator, maximum likelihood (ML) estimator, percentile (PC) estimator, least squares (LS) estimator and weighted least squares (WLS) estimator. Analytical expressions are derived for the bias and the mean squared error. Simulation studies and real data applications show that the ML estimator performs better than others.

Keywords: Uniform minimum variance unbiased estimator; Maximum likelihood estimator; Least squares estimator; Weight least squares estimator; Percentile estimator; Model selection criteria; Exponentiated Gumbel distribution.
\end{abstract}

2000 Mathematics Subject Classification: 62F10.

\section{Introduction}

The exponentiated Gumbel distribution has received considerable interest. Some recent applications of it have included: climate modeling, Nadarajah (2005); stress strength modeling, Kakade et al. (2008); estimation of return values for significant wave height in oceanography (Persson and Rydèn (2010)); modeling of failure times of the air conditioning system of an aeroplane, Raja and Mir (2011); modeling of the runs scored by a cricketer in twenty seven innings at national level, Raja and Mir (2011).

Nadarajah (2006) proposed the exponentiated Gumbel (EG) distribution as a generalization of the classical Gumbel distribution. Its cumulative distribution function (CDF) is specified by

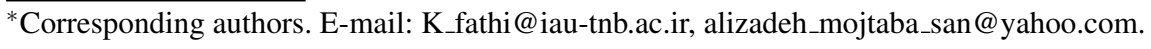




$$
F(x)=\left[e^{-e^{-\frac{x-\alpha}{\beta}}}\right]^{\lambda}
$$

where $x>0, \alpha, \beta, \lambda>0$. The corresponding probability density function (pdf) is

$$
f(x)=\frac{\lambda}{\beta} e^{-\frac{x-\alpha}{\beta}} e^{-\lambda e^{-\frac{x-\alpha}{\beta}}}, x>0, \alpha, \beta, \lambda>0,
$$

where $\alpha$ is the location parameter, $\beta$ is the scale parameter and $\lambda$ is the shape parameter.

Because of the numerous applications of the EG distribution, we feel the importance to investigate efficient estimation of the pdf and the CDF of the EG distribution. We consider several different estimation methods: uniformly minimum variance unbiased (UMVU) estimation, maximum likelihood (ML) estimation, percentile (PC) estimation, least squares (LS) estimation and weight least squares (WLS) estimation.

Similar studies have appeared in the recent literature for other distributions. For example, Bagheri et al. (2014) derive estimators of the pdf and the CDF of a three-parameter generalized exponential-Poisson distribution when all but its shape parameter are assumed known. Recently Bagheri et al. (2016) have considered Efficient estimation of the PDF and the CDF of the Weibull extension model. Alizadeh et al. (2015a) derive estimators of the pdf and the CDF of a threeparameter exponentiated Weibull distribution when all but its shape parameter are assumed known. Also Alizadeh et al. (2015b) derive estimators of the pdf and the CDF of a two-parameter the generalized exponential distribution.

The paper is organized as follows. The MLE and the UMVUE of the pdf and the CDF and their mean squared errors (MSEs) are derived in Sections 2 and 3. The PCEs of the pdf and the CDF are discussed in Section 4. The LSEs and WLSEs of the pdf and the CDF are discussed in Section 5. The estimators are compared by simulation and real data application in Sections 6 and 7. Finally, some discussion on the possible use of the results in the paper is provided in Section 8.

Throughout the paper (except for Section 7), we assume $\lambda$ is unknown, but both $\alpha$ and $\beta$ are known. This assumption is not entirely unreasonable. For example, in the case of the normal distribution and its extensions, the location and scale parameters can be set equal to sample mean and sample standard deviation, respectively. In other words, the data can be standardized before the fitting of a distribution so that its location and scale parameters take "standard" and known values. Standardization of data is common practice.

A future work is to extend the results of the paper to the case that all three parameters of the EG distribution are unknown. There has been work in the literature where the pdf and the CDF have been estimated when all their parameters are unknown.

Such estimation of pdfs is considered by Lech and Maryana (2009) for a trapezoidal distribution, by Koenker and Mizera (2010) for a log-concave distribution, and by Duval (2013) for a compound Poisson distribution. See also Er (1998). Koenker and Mizera (2010) use MLEs.

Estimation of CDFs is considered by Przybilla et al. (2013) for a three-parameter Weibull distribution, by Durot et al. (2013) for a convex discrete distribution, and by Dattner and Reiser (2013) for distributions in measurement error models. Przybilla et al. (2013) use MLEs and Durot et al. (2013) use LSEs. 


\section{Maximum Likelihood Estimator of pdf and CDF}

Let $X_{1}, \ldots, X_{n}$ be a random sample of size $\mathrm{n}$ from the EG distribution. The MLE of $\lambda$ say $\widetilde{\lambda}$ is

$$
\tilde{\lambda}=\frac{n}{\sum_{i=1}^{n} e^{-\frac{x_{i}-\alpha}{\beta}}} .
$$

Therefore, we obtain MLEs of the pdf and the CDF as

$$
\widetilde{f}(x)=\frac{\widetilde{\lambda}}{\beta} e^{-\frac{x-\alpha}{\beta}} e^{-\widetilde{\lambda} e^{-\frac{x-\alpha}{\beta}}}
$$

and

$$
\widetilde{F}(x)=\left[e^{-e^{-\frac{x-\alpha}{\beta}}}\right]^{\tilde{\lambda}}
$$

respectively, for $x>0, \alpha, \beta>0$. After some elementary algebra, we obtain the pdf of $\tilde{\lambda}=s$ as

$$
g(s)=\frac{n^{n} \lambda^{n}}{\Gamma(n)} \frac{e^{-\frac{n \lambda}{s}}}{s^{n+1}}
$$

for $s>0$.

In the following, we calculate $E\left(\widetilde{f}(x)^{r}\right)$ and $E\left(\widetilde{F}(x)^{r}\right)$.

Theorem 2.1. The $E\left(\widetilde{f}(x)^{r}\right)$ and $E\left(\widetilde{F}(x)^{r}\right)$ are given, respectively, as

$$
\begin{gathered}
E\left(\widetilde{f}(x)^{r}\right)=2 \frac{(n \lambda)^{\frac{r+n}{2}} e^{-r \frac{x-\alpha}{\beta}}}{\Gamma(n) \beta^{r}}\left(\frac{1}{r e^{-\frac{x-\alpha}{\beta}}}\right)^{\frac{r-n}{2}} K_{r-n}\left(2 \sqrt{n \lambda r e^{-\frac{x-\alpha}{\beta}}}\right) . \\
E\left(\widetilde{F}(x)^{r}\right)=2 \frac{(n \lambda)^{\frac{n}{2}}}{\Gamma(n)}\left(\frac{1}{r e^{-\frac{x-\alpha}{\beta}}}\right)^{\frac{-n}{2}} K_{-n}\left(2 \sqrt{n \lambda r e^{-\frac{x-\alpha}{\beta}}}\right)
\end{gathered}
$$

and $K_{v}(\cdot)$ denotes the modified Bessel function of the second kind of order $v$.

Proof. For more details see Alizadeh et al. (2015b).

It must be note that the estimators, $\widetilde{f}(x)$ and $\widetilde{F}(x)$, are biased for $f(x)$ and $F(x)$, respectively. In the following theorem we obtain the MSE of $\widetilde{f}(x)$ and $\widetilde{F}(x)$.

\section{Theorem 2.2.}

$$
\text { (A) } \begin{aligned}
M S E(\widetilde{f}(x)) & =\frac{2(n \lambda)^{\frac{2+n}{2}} e^{-2 \frac{x-\alpha}{\beta}}}{\Gamma(n) \beta^{2}}\left(\frac{1}{2 e^{-\frac{x-\alpha}{\beta}}}\right)^{\frac{2-n}{2}} K_{2-n}\left(2 \sqrt{2 n \lambda e^{-\frac{x-\alpha}{\beta}}}\right) \\
& -\frac{4 f(x)(n \lambda)^{\frac{1+n}{2}} e^{-\frac{x-\alpha}{\beta}}}{\Gamma(n) \beta}\left(\frac{1}{e^{-\frac{x-\alpha}{\beta}}}\right)^{\frac{1-n}{2}} K_{1-n}\left(2 \sqrt{n \lambda e^{-\frac{x-\alpha}{\beta}}}\right)-f^{2}(x) .
\end{aligned}
$$




$$
\text { (B) } \begin{aligned}
\operatorname{MSE}(\widetilde{F}(x)) & =\frac{2(n \lambda)^{\frac{n}{2}}}{\Gamma(n)}\left(\frac{1}{2 e^{-\frac{x-\alpha}{\beta}}}\right)^{\frac{-n}{2}} K_{-n}\left(2 \sqrt{2 n \lambda e^{-\frac{x-\alpha}{\beta}}}\right) \\
& -\frac{4 F(x)(n \lambda)^{\frac{n}{2}}}{\Gamma(n)}\left(\frac{1}{r e^{-\frac{x-\alpha}{\beta}}}\right)^{\frac{-n}{2}} K_{-n}\left(2 \sqrt{n \lambda r e^{-\frac{x-\alpha}{\beta}}}\right)-F^{2}(x)
\end{aligned}
$$

Proof. Note that $\operatorname{MSE}(\widetilde{f}(x))=E(\widetilde{f}(x))^{2}-2 f(x) E(\widetilde{f}(x))+f^{2}(x)$. We have an expression for $E(\widetilde{f}(x))$ from Theorem 2.1. An expression for $E(\widetilde{f}(x))^{2}$ can be obtained similarly by following the proof of Theorem 2.1, yielding the given expression for $\operatorname{MSE}(\widetilde{f}(x))$. The proof for $\operatorname{MSE}(\widetilde{F}(x))$ is similar.

It is clear that the ML estimator of $\lambda$ is biased and $\operatorname{MSE}(\widetilde{\lambda})=\frac{(n+2) \lambda^{2}}{(n-1)(n-2)}$.

\section{UMVU Estimator of pdf and CDF}

In this section, we find the UMVU estimators of the pdf and the CDF and their MSEs.

Let $X_{1}, \ldots, X_{n}$ be a random sample of size $\mathrm{n}$ from the EG distribution is given by (1.2). Then $T=\sum_{i=1}^{n} e^{-\frac{x_{i}-\alpha}{\beta}}$ is a complete sufficient statistic for the unknown parameter $\lambda$ (when both $\alpha$ and $\beta$ are known) and the pdf of $\mathrm{T}$ is as

$$
h^{*}(t)=\frac{\lambda^{n}}{\Gamma(n)} t^{n-1} e^{-\lambda t}
$$

for $t>0$. According to Lehmann Scheffe theorem if $h\left(x_{1} \mid t\right)=f^{*}(t)$ is the conditional pdf of $X_{1}$ given $T$, we have

$$
E\left[f^{*}(T)\right]=\int h\left(x_{1} \mid t\right) h^{*}(t) d t=\int h\left(x_{1}, t\right) d t=f\left(x_{1}\right),
$$

where $h\left(x_{1}, t\right)$ is the joint pdf of $X_{1}$ and $T$. Therefore $f^{*}(t)$ is the UMVUE of $f(x)$.

Lemma 3.1. The joint distribution of $X_{1}$ and $T$ is as

$$
h\left(x_{1}, t\right)=\frac{\lambda}{\beta} e^{-\frac{x_{1}-\alpha}{\beta}} e^{-\lambda e^{-\frac{x_{1}-\alpha}{\beta}}} \frac{\lambda^{n-1}}{\Gamma(n-1)}(t-k)^{n-2} e^{-\lambda(t-k)}, k<t<\infty,
$$

where $k=e^{-\frac{x_{1}-\alpha}{\beta}}$.

Proof. We have the joint distribution of $\left(X_{1}, X_{2}, \ldots, X_{n}\right)$ as

$$
f\left(x_{1}, x_{2}, \ldots, x_{n}\right)=\prod_{i=1}^{n} f\left(x_{i}\right)=\prod_{i=1}^{n}\left(\frac{\lambda}{\beta} e^{-\frac{x_{i}-\alpha}{\beta}} e^{-\lambda e^{-\frac{x_{i}-\alpha}{\beta}}}\right) .
$$

In order to find the joint pdf of $\left(X_{1}, T\right)$, we apply the transformation: 
$y_{1}=e^{-\frac{x_{1}-\alpha}{\beta}}, y_{2}=e^{-\frac{x_{2}-\alpha}{\beta}}, \ldots y_{n-1}=e^{-\frac{x_{n-1}-\alpha}{\beta}}, t=\sum_{i=1}^{n} e^{-\frac{x_{i}-\alpha}{\beta}}$. Then by using some elementary algebra and $(n-2)$ integrations for $y_{2}, y_{3}, \ldots, y_{n-1}$, the proof is done.

Theorem 3.1. Let $T=t$ be given. Then

$$
\widehat{f}(x)=\frac{(n-1) e^{-\frac{x_{1}-\alpha}{\beta}}}{\beta} \frac{(t-k)^{n-2}}{t^{n-1}},
$$

is a UMVUE for $f(x)$, and

$$
\widehat{F}(x)=\left[1-\frac{e^{-\frac{x_{1}-\alpha}{\beta}}}{t}\right]^{n-1}
$$

is a UMVUE for $F(x)$, where $k=e^{-\frac{\left(x_{1}-\alpha\right)}{\beta}}$ and $k<t<\infty$.

Proof. For more details see Alizadeh et al. (2015b).

Theorem 3.2. The MSEs of $\widehat{f}(x)$ and $\widehat{F}(x)$ are given by

$$
\operatorname{MSE}(\widehat{f}(x))=\frac{(n-1)^{2}}{\beta^{2}} e^{-2 \frac{x-\alpha}{\beta}} \frac{\lambda^{n}}{\Gamma(n)} \sum_{i=0}^{2 n-4}(-k)^{i}\left(\begin{array}{c}
2 n-4 \\
i
\end{array}\right) \Gamma(n-2-i, \lambda k)-f^{2}(x)
$$

and

$$
\operatorname{MSE}(\widehat{F}(x))=\frac{\lambda^{n}}{\Gamma(n)} \sum_{i=0}^{2 n-2}(-k)^{i}\left(\begin{array}{c}
2 n-2 \\
i
\end{array}\right) \Gamma(n-i, \lambda k)-F^{2}(x)
$$

where $\Gamma(s, x)=\int_{x}^{\infty} t^{s-1} e^{-t} d t$ denotes the complementary incomplete gamma function.

Proof. For more details see Alizadeh et al. (2015b).

It must be note that UMVU estimator of $\lambda$ is $\widehat{\lambda}=\frac{n-1}{\sum_{i=1}^{n} e^{-\frac{x_{i}-\alpha}{\beta}}}$ and $\operatorname{MSE}(\widehat{\lambda})=\frac{\lambda^{2}}{n-2}$.

In the following section we present other estimators.

\section{Estimators Based on Percentiles}

Estimation based on percentiles was originally explored by Kao (1959), see also Mann et al. (1974) and Johnson et al. (1994). Percentiles estimators are based on inverting the CDF. Since the EG has a closed form $\mathrm{CDF}$, its parameters can be obtained estimated using percentiles.

Let $X_{1}, \ldots, X_{n}$ denote a random sample from the EG distribution and let $X_{(1)}<\cdots<X_{(n)}$ denote the ordered sample. Also let $p_{i}=i /(n+1)$. The percentile estimator of $\lambda$ (when $\alpha$ and $\beta$ are known ), say $\widetilde{\lambda}_{p c}$, is the value minimizing $\sum_{i=1}^{n}\left(p_{i}+\lambda e^{-\frac{x_{(i)}-\alpha}{\beta}}\right)^{2}$. So, the percentile estimators of the pdf and the CDF are

$$
\begin{aligned}
& \widetilde{f}_{p c}(x)=\frac{\widetilde{\lambda}_{p c}}{\beta} e^{-\frac{x-\alpha}{\beta}} e^{-\widetilde{\lambda}_{p c} e^{-\frac{x-\alpha}{\beta}}} \\
& \widetilde{F}_{p c}(x)=\left(e^{-e^{-\frac{x-\alpha}{\beta}}}\right)^{\widetilde{\lambda}_{p c}} .
\end{aligned}
$$




\section{Least Squares and Weighted Least Squares Estimators}

In this section, we derive regression based estimators of the unknown parameter. This method was originally suggested by Swain et al. (1988) to estimate the parameters of beta distributions. It can be used some other cases also. Suppose $X_{1}, \ldots, X_{n}$ is a random sample of size $n$ from a CDF $F(\cdot)$ and suppose $X_{(i)}, i=1, \ldots, n$ denote the ordered sample. The proposed method uses the CDF of $F\left(X_{(i)}\right)$. For a sample of size $n$, we have

$$
E\left[F\left(X_{(j)}\right)\right]=\frac{j}{n+1}, \operatorname{Var}\left[F\left(X_{(j)}\right)\right]=\frac{j(n-j+1)}{(n+1)^{2}(n+2)}, \operatorname{Cov}\left[F\left(Y_{(j)}\right), F\left(X_{(k)}\right)\right]=\frac{j(n-k+1)}{(n+1)^{2}(n+2)} \text { for } j<k,
$$
see Johnson et al. (1994). Using the expectations and the variances, two variants of the least squares method follow.

\subsection{Method 1: Least Squares Estimators}

This method is based on minimizing $\sum_{j=1}^{n}\left[F\left(X_{(j)}\right)-\frac{j}{n+1}\right]^{2}$, with respect to the unknown parameters. In case of EG distribution the least squares estimators of $\lambda$ (when $\alpha$ and $\beta$ are known ), say $\tilde{\lambda}_{l s}$, is the value minimizing $\sum_{j=1}^{n}\left(e^{-\lambda e^{-\frac{x_{(j)}-\alpha}{\beta}}}-\frac{j}{n+1}\right)^{2}$. So, the LS estimators the pdf and the CDF are

$$
\begin{aligned}
& \widetilde{f}_{l s}(x)=\frac{\widetilde{\lambda}_{l s}}{\beta} e^{-\frac{x-\alpha}{\beta}} e^{-\widetilde{\lambda}_{l s} e^{-\frac{x-\alpha}{\beta}}} \\
& \widetilde{F}_{l s}(x)=\left(e^{-e^{-\frac{x-\alpha}{\beta}}}\right)^{\tilde{\lambda}_{l s}} .
\end{aligned}
$$

It is difficult to find the expectation and the MSE of these estimators by mathematical methods. We can calculate them by means of a simulation study.

\subsection{Method 2: Weighted Least Squares Estimators}

This method is based on minimizing

$$
\sum_{j=1}^{n} w_{j}\left[F\left(X_{(j)}\right)-\frac{j}{n+1}\right]^{2} \text {, with respect to the unknown parameters, where } w_{j}=\frac{1}{\operatorname{Var}\left(F\left(Y_{(j)}\right)\right)}=
$$
$\frac{(n+1)^{2}(n+2)}{j(n-j-1)}$. In case of EG distribution the least squares estimators of $\lambda$ (when $\alpha$ and $\beta$ are known), say $\tilde{\lambda}_{w l s}$, is the value minimizing $\sum_{j=1}^{n} w_{j}\left(e^{-\lambda e^{-\frac{x_{(i)}-\alpha}{\beta}}}-\frac{j}{n+1}\right)^{2}$, with respect to $\lambda$. So, the WLS estimators of the pdf and the CDF are

$$
\begin{aligned}
& \widetilde{f}_{w l s}(x)=\frac{\tilde{\lambda}_{w l s}}{\beta} e^{-\frac{x-\alpha}{\beta}} e^{-\tilde{\lambda}_{w l s} e^{-\frac{x-\alpha}{\beta}}} \\
& \widetilde{F}_{w l s}(x)=\left(e^{-e^{-\frac{x-\alpha}{\beta}}}\right)^{\widetilde{\lambda}_{w l s}} .
\end{aligned}
$$

It is difficult to find the expectation and the MSE of these estimators by mathematical methods. We can calculate them by means of a simulation study.

\section{Simulation study}

Here, we perform a simulation study to compare the performances of the following estimators: MLE, UMVUE, PCE, LSE and WLSE of the pdf and the CDF. The comparison is based on MSEs. 
The MSEs were computed by generating one thousand replications of samples of size $n=5, \ldots, 100$ from the EG distribution with $(\alpha, \beta, \lambda)=(0.5,0.5,0.5),(2,2,2),(3,3,3),(1,2,3),(3,2,1),(2,1,1)$. Figures 1 to 2 plot the derivation of the MSEs of the UMVUE, WLSE, LSE, and the PCE from the MSE of the MLE versus n.

We can see from the figures that the ML estimators of the and the CDF are the most efficient for all $n$. The UMVU estimators are the second most efficient for all $n$. The WLS estimators are third most efficient for all $n$. The LS estimators are the fourth most efficient for all $n$. The PC estimators are the least efficient for all $n$. We can also see that the gain in efficiency by using the MLE over others increases with increasing $\lambda$.

\section{Data analysis}

Here, we use a real data set to compare the performances of MLE, PCE, LSE and WLSE of the pdf and the CDF. The first data set represents the strength data originally reported in Badar and Priest (1982). It represents the strength measured in GPA for single carbon fibers and impregnated 1000-carbon fiber tows. Single fibers were tested under tension at gauge length of $10 \mathrm{~mm}$. They are as follows:

$1.901,2.132,2.203,2.228,2.257,2.350,2.361,2.396,2.397,2.445,2.454,2.474,2.518,2.522$, $2.525,2.532,2.575,2.614,2.616,2.618,2.624,2.659,2.675,2.738,2.740,2.856,2.917,2.928$, $2.937,2.937,2.977,2.996,3.030,3.125,3.139,3.145,3.220,3.223,3.235,3.243,3.264,3.272$, $3.294,3.332$, 3.346, 3.377, 3.408, 3.435, 3.493, 3.501, 3.537, 3.554, 3.562, 3.628, 3.852, 3.871, $3.886,3.971,4.024,4.027,4.225,4.395,5.020$.

When one works with real data, all of the parameters, $\alpha, \beta$ and $\lambda$ are unknown. Therefore we use the following equations for estimating unknown parameters under different methods.

Let $X_{1}, \ldots, X_{n}$ be a random sample of size $\mathrm{n}$ from the EG distribution given by (1.2), then the log-likelihood function of the observed sample is

$$
L(\alpha, \beta, \lambda)=n \log \lambda-n \log \beta-\sum_{i=1}^{n} \frac{x_{i}-\alpha}{\beta}-\lambda \sum_{i=1}^{n} e^{-\frac{x_{i}-\alpha}{\beta}} .
$$

The MLEs of $\alpha, \beta$ and $\lambda$, say $\widetilde{\alpha}, \widetilde{\beta}$ and $\widetilde{\lambda}$ respectively, can be obtained as the solutions of the equations

$$
\begin{aligned}
& \frac{\partial L}{\partial \lambda}=\frac{n}{\lambda}-\sum_{i=1}^{n} e^{-\frac{x_{i}-\alpha}{\beta}}=0, \\
& \frac{\partial L}{\partial \beta}=-\frac{n}{\beta}+\sum_{i=1}^{n} \frac{x_{i}-\alpha}{\beta^{2}}-\lambda \sum_{i=1}^{n} \frac{x_{i}-\alpha}{\beta^{2}} e^{-\frac{x_{i}-\alpha}{\beta}}=0, \\
& \frac{\partial L}{\partial \alpha}=\frac{n}{\beta}-\frac{\lambda}{\beta} \sum_{i=1}^{n} e^{-\frac{x_{i}-\alpha}{\beta}}=0 .
\end{aligned}
$$

The PCEs of $\alpha, \beta$ and $\lambda$, say $\widetilde{\alpha}_{p c}, \widetilde{\beta}_{p c}$ and $\widetilde{\lambda}_{p c}$ respectively, can be obtained by minimizing $\sum_{j=1}^{n}\left[p_{j}+\lambda e^{-\frac{x_{(j)}-\alpha}{\beta}}\right]^{2}$.

The LSEs of $\alpha, \beta$ and $\lambda$, say $\widetilde{\alpha}_{l s}, \widetilde{\beta}_{l s}$ and $\widetilde{\lambda}_{l s}$ respectively, can be obtained by minimizing $\sum_{j=1}^{n}\left[e^{-\lambda e^{-\frac{x_{(j)}-\alpha}{\beta}}}-\frac{j}{n+1}\right]^{2}$. 
The weighted least squares estimators of the unknown parameters, $\alpha, \beta$ and $\lambda$, say $\widetilde{\alpha}_{w l s}, \widetilde{\beta}_{w l s}$ and $\widetilde{\lambda}_{w l s}$ respectively, can be obtained by minimizing $\sum_{j=1}^{n} w_{j}\left[e^{-\lambda e^{-\frac{x_{(j)}-\alpha}{\beta}}}-\frac{j}{n+1}\right]^{2}$, where $w_{j}=$ $\frac{1}{\operatorname{Var}\left(F\left(Y_{(j)}\right)\right)}=\frac{(n+1)^{2}(n+2)}{j(n-j+1)}$.

The EG distribution was fitted to the strength data by MLE, PCE, LSE and WLSE. Table 1 gives the estimates of $\alpha, \beta, \lambda$ and the corresponding log-values. The log-likelihood value is the largest for the MLE.

Table 1. Estimates of the parameters and the corresponding log-likelihood.

\begin{tabular}{ccccc}
\hline & Estimate of $\alpha$ & Estimate of $\beta$ & Estimate of $\lambda$ & Log-Likelihood \\
\hline MLE & 2.4235807 & 0.5125927 & 1.9638011 & -56.51386 \\
PCE & 2.4213873 & 0.5196643 & 1.9632186 & -56.52364 \\
LSE & 2.3885197 & 0.5672428 & 1.9540527 & -57.07471 \\
WLSE & 2.4040106 & 0.5433652 & 1.9584667 & -56.70443 \\
\hline
\end{tabular}

Table 2. Mean absolute and mean squared deviations based on the Q-Q plots.

\begin{tabular}{ccc}
\hline & MAD & MSD \\
\hline MLE & 0.5003341 & 0.4144542 \\
LSE & 0.5536773 & 0.5075393 \\
WLSE & 0.5303706 & 0.4657096 \\
PCE & 0.5072366 & 0.4259684 \\
\hline
\end{tabular}

Figures 4, 5 and 6 show the Q-Q plots (observed quantiles versus expected quantiles), the density plots (fitted PDFs versus empirical histogram) and the P-P plots (observed probabilities versus expected probabilities) for the four different estimation methods. Visual inspection of these figures shows that the ML estimator provides the best fit. To verify this observation, we used mean absolute deviations (MADs) and mean squared deviations (MSDs) to quantify the amount of discrepancy between the observed and expected. The MADs and MSDs between the observed and expected quantiles for the four different estimation methods are shown in Table 2. These tables shows that the values of MAD and MSD are smallest for the ML estimator.

We also compared the estimation methods by means of model selection criteria. The ones we considered are: pure' maximum likelihood (ML) $=-2 \ln L(\theta)$, Akaike information criterion (AIC) $=-2 \ln L(\theta)+2 k$, corrected AIC (AICc) $=-2 \ln L(\theta)+2 k \frac{n}{n-k-1}$, Bayes information criterion (BIC, also known as Schwarz criterion $)=-2 \ln L(\theta)+k \ln n$, Hannan-Quinn criterion $(\mathrm{HQC})=-2 \ln L(\theta)+$ $2 k \ln \ln n$,

where $\ln L(\theta)$ denotes the $\log$-likelihood, $n$ denotes the number of observations (i.e., the length of $x$ ) and $k$ denotes the number of parameters of the distribution. The smaller the values of these criteria the better the fit. For more discussion on these criteria, see Burnham and Anderson (2004) and Fang (2011).

Table 3 gives values of the model selection criteria for the four different estimation methods. We can see that the ML estimators give the smallest values for all five model selection criteria. 

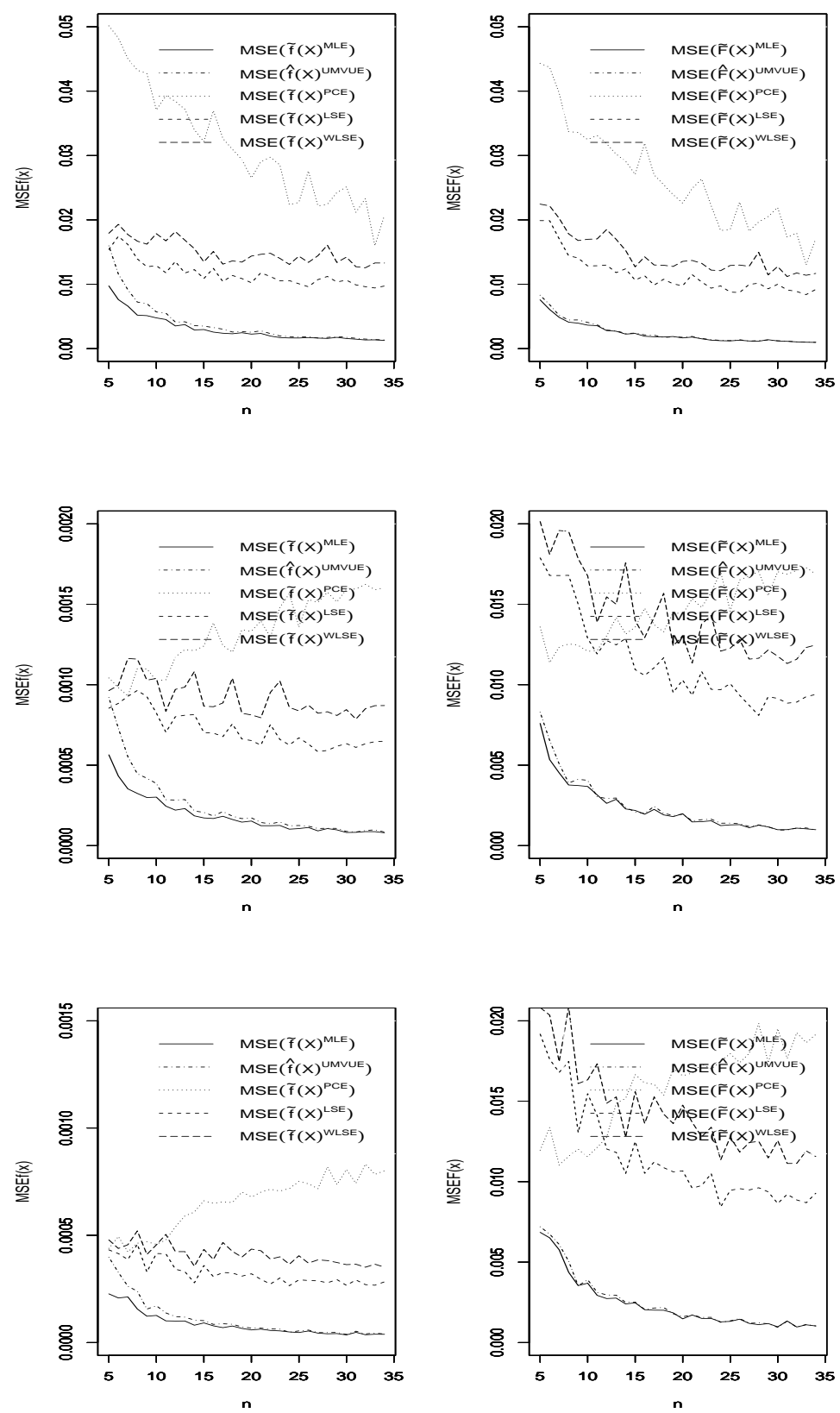

Fig. 1. MSEs of MLE, UMVUE, PCE, LSE and WLSE for $f(x)$ and $(\alpha, \beta, \lambda)=(0.5,0.5,0.5)$ (top left), $F(x)$ and $(\alpha, \beta, \lambda)=(0.5,0.5,0.5)$ (top right), $f(x)$ and $(\alpha, \beta, \lambda)=(2,2,2)$ (middle left), $F(x)$ and $(\alpha, \beta, \lambda)=(2,2,2)$ (middle right), $f(x)$ and $(\alpha, \beta, \lambda)=(3,3,3)$ (bottom left) and $F(x)$ and $(\alpha, \beta, \lambda)=(3,3,3)$ (bottom right).

Hence, evidence based on the MSEs in the simulation study, the log-likelihood values, the Q-Q plots, the density plots, the distribution plots and the model selection criteria show that the ML estimators for the pdf and the CDF are the best. 

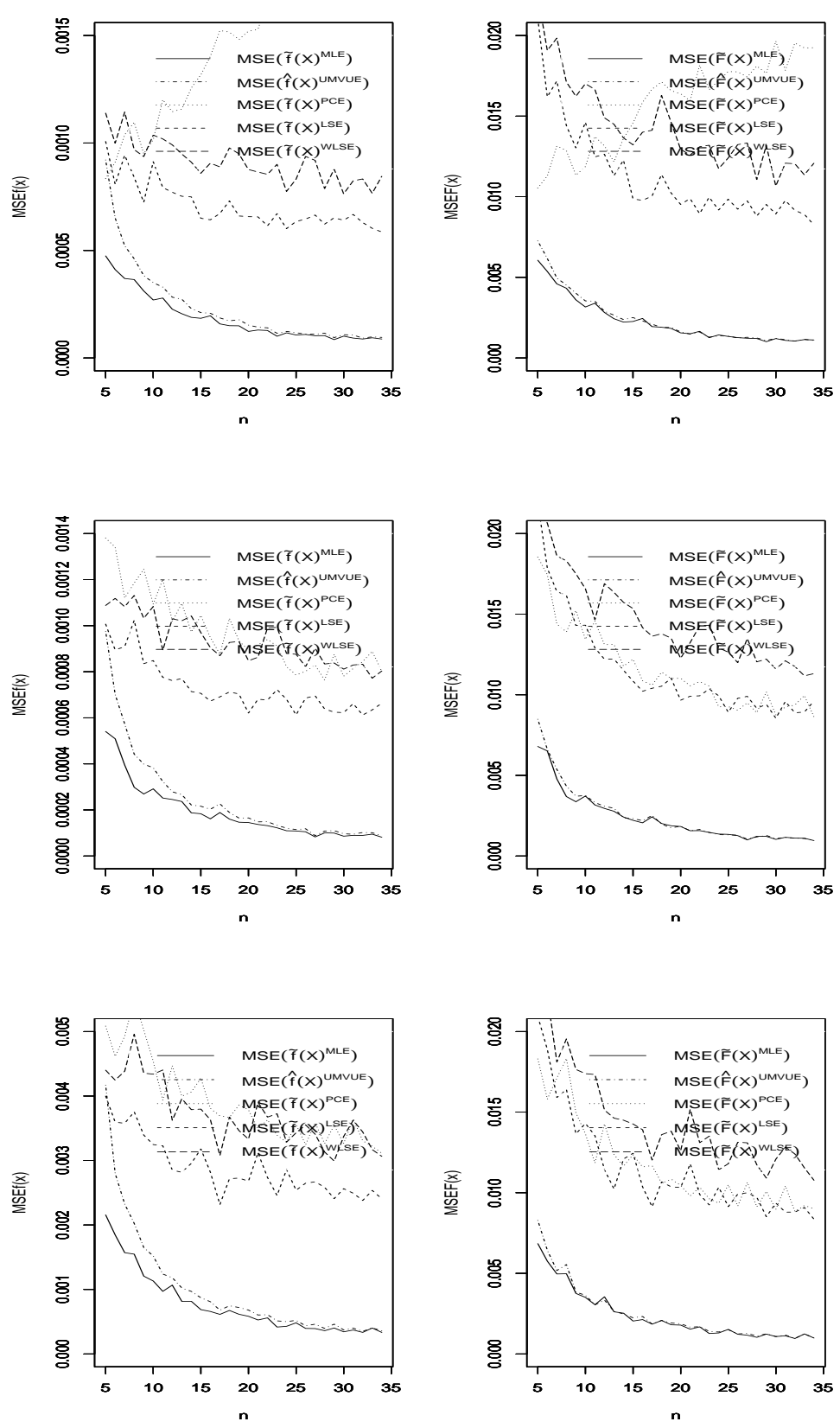

Fig. 2. MSEs of MLE, UMVUE, PCE, LSE and WLSE for $f(x)$ and $(\alpha, \beta, \lambda)=(1,2,3)$ (top left), $F(x)$ and $(\alpha, \beta, \lambda)=$ $(1,2,3)$ (top right), $f(x)$ and $(\alpha, \beta, \lambda)=(3,2,1)$ (middle left), $F(x)$ and $(\alpha, \beta, \lambda)=(3,2,1)$ (middle right), $f(x)$ and $(\alpha, \beta, \lambda)=(2,1,1)$ (bottom left) and $F(x)$ and $(\alpha, \beta, \lambda)=(2,1,1)$ (bottom right).

\section{Discussion}

We have compared five different estimators (the UMVU estimator, the ML estimator, the PC estimator, the LS estimator and WLS estimator) for the pdf and the CDF of the EG distribution when 

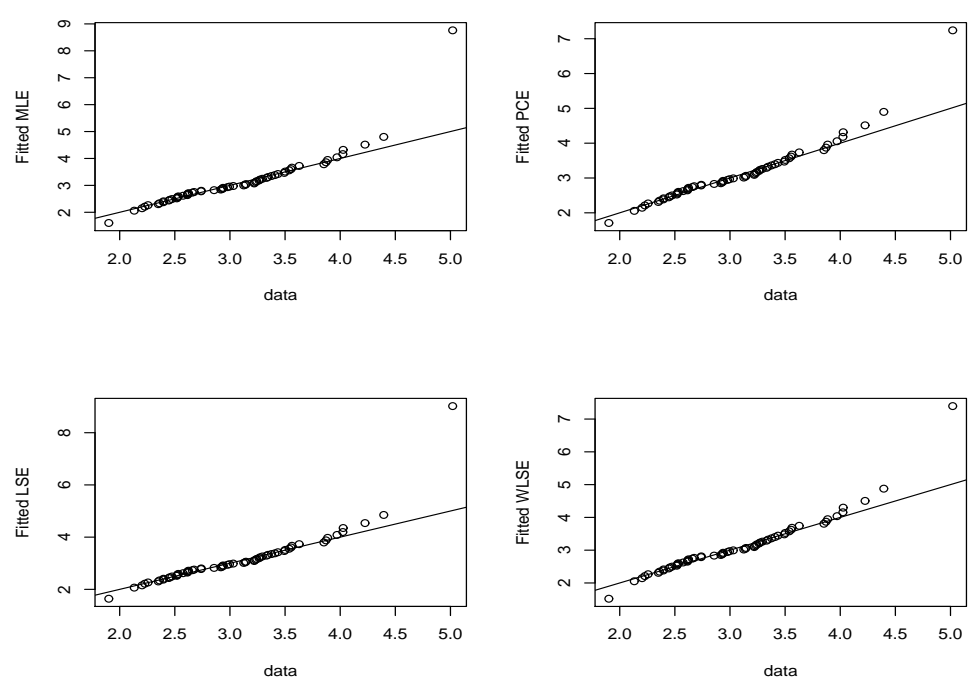

Fig. 3. Q-Q plots for the fit of the four different estimation methods.

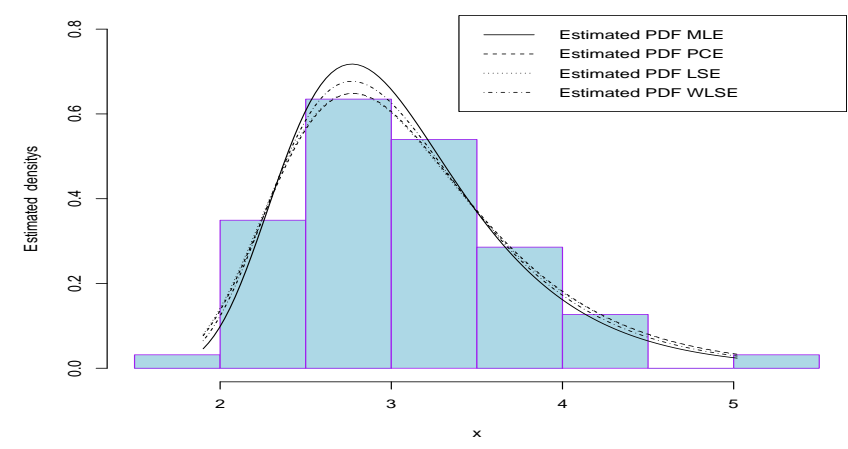

Fig. 4. Fitted pdfs versus the histogram for the four different estimation methods.

Table 3. The values of model selection criteria for waiting time data.

\begin{tabular}{cccccc}
\hline & ML & AIC & BIC & AICc & HQC \\
\hline MLE & 113.0277 & 119.0277 & 125.4571 & 119.4345 & 121.5564 \\
PCE & 113.0473 & 119.0473 & 125.4767 & 119.4541 & 121.5760 \\
LSE & 114.1494 & 120.1494 & 126.5788 & 120.5562 & 122.6781 \\
WLSE & 113.4089 & 119.4089 & 125.8383 & 119.8156 & 121.9376 \\
\hline
\end{tabular}

the location and scale parameters are assumed to be known. Explicit expressions are given for the MSEs of the UMVU and ML estimators.

We have compared the performances of the five estimators by simulation and a real data application. The results show that the ML estimator performs the best in terms of the MSEs in the 

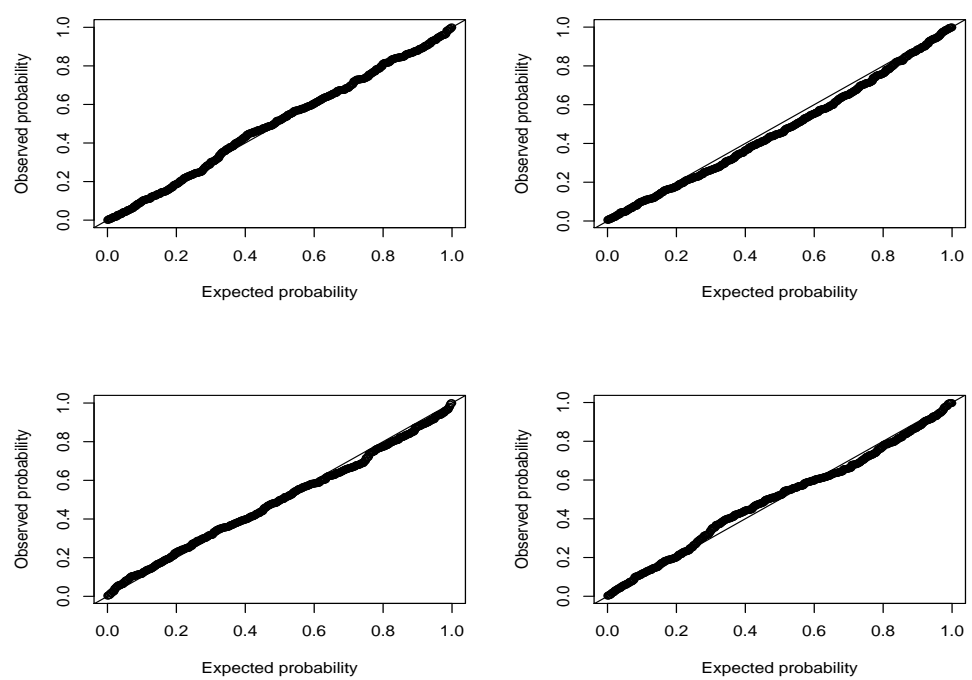

Fig. 5. Fitted CDFs versus the empirical CDF for the four different estimation methods.

simulation study, the log-likelihood values, the Q-Q plots, the mean absolute and mean squared deviations based on the Q-Q plots, the density plots, the P-P plots, AIC, AICc, BIC and HQC.

Comparisons of the kind performed can be useful to find the best estimators for the pdf and the CDF. The best estimators for the pdf can be used to estimate functionals of the pdf like

- the differential entropy of $f$ defined by

$$
-\int_{-\infty}^{\infty} f(x) \log f(x) d x
$$

- the Rényi entropy defined by

$$
\frac{1}{1-\gamma} \log \int_{0}^{\infty} f^{\gamma}(x) d x
$$

for $\gamma>0$ and $\gamma \neq 1$;

- the Kulback-Liebler divergence of $f$ from an arbitrary pdf $f_{0}$ defined by

$$
\int_{-\infty}^{\infty} f(x) \ln \left\{f(x) / f_{0}(x)\right\} d x
$$

- the Fisher information defined by

$$
\int_{-\infty}^{\infty}\left[\frac{\partial}{\partial \theta} f(x ; \theta)\right]^{2} f(x ; \theta) d x,
$$

where $\theta$ is a parameter specifying the pdf.

Estimation of differential entropy is considered by Nilsson and Kleijn (2007) for data located on embedded manifolds and by Hampel (2008) for positive random variables. The latter paper illustrates an application in computational neuroscience. Estimation of negentropy is considered by Dejak et al. (1993) for time series models. This paper illustrates an application to environmental 
data. Estimation of Rényi entropy is considered by Kayal et al. (2013) for exponential distributions. UMVU and ML estimators are given. Estimation of Fisher information is considered by Mielniczuk and Wojtys (2010) for model selection, and by Li et al. (2004).

The best estimators for the CDF can be used to estimate functionals of the CDF like

- cumulative residual entropy of $F$ defined by

$$
\int_{0}^{\infty}[1-F(\lambda)+F(-\lambda)] \log [1-F(\lambda)+F(-\lambda)] d \lambda ;
$$

- the quantile function of $F$ defined by $F^{-1}(\cdot)$;

- the Bonferroni curve defined by

$$
\frac{1}{p \mu} \int_{0}^{p} F^{-1}(t) d t
$$

where $\mu=E(X)$;

Estimation of cumulative residual entropy is considered by Bratpvrbajgyran [30] for the Rayleigh distribution. Estimation of quantiles is considered by Ehsanes Saleh et al. (1988) for a location-scale family of distributions including the Pareto, exponential and double exponential distributions, by Ehsanes Saleh et al. (1983) for the normal distribution, by Rojo (1998) for an increasing failure rate distribution. Estimation of the Lorenz curve is considered by Woo and Yoon (2001) for a Pareto distribution. See also Gastwirth (1972).

The best estimators for both the pdf and the CDF can be used to estimate functionals of the pdf and the CDF like

- probability weighted moments defined by

$$
\int_{-\infty}^{\infty} x F^{r}(x) f(x) d x
$$

- the hazard rate function defined by

$$
\frac{f(x)}{1-F(x)}
$$

- the reverse hazard rate function defined by

$$
\frac{f(x)}{F(x)}
$$

Unbiased estimation of probability weighted moments is considered by Wang (1990). Estimation of hazard rate functions is considered by Saunders and Myhre (1983) for two-parameter decreasing hazard rate distributions, by Hsieh (1990) for the inverse Gaussian distribution, by Lin et al. (2003) for the linear hazard rate distribution, and by Ahn et al. (2007) for a mixture distribution with censored lifetimes. Saunders and Myhre (1983) use MLEs, Hsieh (1990) uses MLEs and UMVUEs, Lin et al. (2003) use MLEs obtained via the EM algorithm, and Ahn et al. (2007) use MLEs and a Bayesian approach. Estimation of mean deviation is considered by Hartley (1945) for the normal distribution and by Suzuki (1965) for the Pearson type distribution. The estimators given by the latter are consistent. 


\section{Acknowledgments}

The authors would like to thank the referees and the Editor for careful reading and for comments which greatly improved the paper.

\section{References}

[1] A.K.Md. Ehsanes Saleh, K.M. Hassanein and M. Masoom Ali, Estimation and testing of hypotheses about the quantile function of the normal distribution, J. of Information and Optimization Sciences, 9 (1988) 85-98.

[2] A.K.Md. Ehsanes Saleh, M. Masoom Ali and D. Umbach, Estimating the quantile function of a location-scale family of distributions based on few selected order statistics, J. of Statistical Planning and Inference, 8 (1983) 75-86.

[3] C. Dejak, D. Franco, R. Pastres, G. Pecenik and C. Solidoro, An informational approach to model time series of environmental data through negentropy estimation, Ecological Modelling, 67 (1993) 199-220.

[4] C. Durot, S. Huet, F. Koladjo and S. Robin, Least-squares estimation of a convex discrete distribution, Computational Statistics and Data Analysis, 67 (2013) 282-298.

[5] C. Duval, Density estimation for compound Poisson processes from discrete data, Stochastic Processes and Their Applications, 123 (2013) 3963-3986.

[6] C. Przybilla, A. Fernandez-Canteli and E. Castillo, Maximum likelihood estimation for the threeparameter Weibull cdf of strength in presence of concurrent flaw populations, J. of the European Ceramic Society, 33 (2013) 1721-1727.

[7] C.S. Kakade, D.T. Shirke, and D. Kundu, Inference for $P(Y<X)$ in exponentiated Gumbel distribution, J. of Statistics and Applications, 3 (2008) 121-133.

[8] C.-T. Lin, S.J.S. Wu and N. Balakrishnan, Parameter estimation for the linear hazard rate distribution based on records and inter-record times, Communications in Statistics-Theory and Methods, 32 (2003) 729-748.

[9] D. Hampel, Estimation of differential entropy for positive random variables and its application in computational neuroscience, in Modeling and Simulation in Science, Engineering and Technology, (2008) 213-224.

[10] G.K. Er, A method for multi-parameter PDF estimation of random variables, Structural Safety, 20 (1998) 25-36.

[11] G. Suzuki, A consistent estimator for the mean deviation of the Pearson type distribution, Annals of the Institute of Statistical Mathematics, 17 (1965) 271-285.

[12] H.K. Hsieh, Estimating the critical time of the inverse Gaussian hazard rate, IEEE Transactions on Reliability, 39 (1990) 342-345.

[13] H.O. Hartley, Note on the calculation of the distribution of the estimate of mean deviation in normal samples, Biometrika, 33 (1945) 257-258.

[14] I. Dattner and B. Reiser, Estimation of distribution functions in measurement error models, J. of Statistical Planning and Inference, 143 (2013) 479-493.

[15] J.H.K. Kao, A graphical estimation of mixed Weibull parameters in life testing electron tubes, Technometrics, 1 (1959) 389-407.

[16] J.L. Gastwirth, The estimation of the Lorenz curve and Gini index, The Review of Economics and Statistics, 54 (1972) 306-316.

[17] J. Mielniczuk and M. Wojtys, Estimation of Fisher information using model selection, Metrika, 72 (2010) 163-187.

[18] J. Rojo, Estimation of the quantile function of an IFRA distribution, Scandinavian Journal of Statistics, 25 (1998) 293-310.

[19] J. Swain, S. Venkatraman and J. Wilson, Least squares estimation of distribution function in Johnson's translation system, J. of Statistical Computation and Simulation, 29 (1988) 271-297.

[20] J.S. Woo and G.E. Yoon, Estimations of Lorenz curve and Gini index in a Pareto distribution, Соттиnications for Statistical Applications and Methods, 8 (2001) 249-256. 
[21] K.P. Burnham and D.R. Anderson, Multimodel inference: Understanding AIC and BIC in model selection, Sociological Methods and Research, 33 (2004) 261-304.

[22] K. Persson and J. Rydén, Exponentiated Gumbel distribution for estimation of return levels of significant wave height, J. of Environmental Statistics, 1 (2010) 1-12.

[23] M. Alizadeh, S.F. Bagheri, E. Baloui Jamkhaneh and S. Nadarajah, Estimates of the PDF and the CDF of the exponentiated Weibull distribution, Brazilian Journal of Probability and Statistics, 29(3) (2015) 695-716.

[24] M. Alizadeh, S. Rezaei, S. F. Bagheri and S. Nadarajah, Efficient estimation for the generalized exponential distribution, Statistical Papers, 56(4) (2015) 1015-1031. DOI: 10.1007/00362-014-0621-7.

[25] M.G. Badar and A.M. Priest, Statistical aspects of fiber and bundle strength in hybrid composites, in Progress in Science and Engineering Composites, editors T. Hayashi, K. Kawata and S. Umekawa, ICCM-IV, Tokyo, (1982) 1129-1136.

[26] M. Nilsson and W.B. Kleijn, On the estimation of differential entropy from data located on embedded manifolds, IEEE Transactions on Information Theory, 53 (2007) 2330-2341.

[27] N.L. Johnson, S. Kotz and N. Balakrishnan, Continuous Univariate Distributions, (volume 1, second edition, New York, Wiley, 1994).

[28] N.R. Mann, R.E. Schafer and N.D. Singpurwalla, Methods for Statistical Analysis of Reliability and Life Data, New York, Wiley, 1974.

[29] Q.J. Wang, Unbiased estimation of probability weighted moments and partial probability weighted moments from systematic and historical flood information and their application to estimating the GEV distribution, J. of Hydrology, 120 (1990) 115-124.

[30] S. Bratpvrbajgyran, A test of goodness of fit for Rayleigh distribution via cumulative residual entropy, in Proceedings of the 8th World Congress in Probability and Statistics.

[31] S.C. Saunders and J.M. Myhre, Maximum likelihood estimation for two-parameter decreasing hazard rate distributions using censored data, J. of the American Statistical Association, 78 (1983) 664-673.

[32] S.E. Ahn, C.S. Park and H.M. Kim, Hazard rate estimation of a mixture model with censored lifetimes, Stochastic Environmental Research and Risk Assessment, 21 (2007) 711-716.

[33] S. Kayal, S. Kumar and P. Vellaisamy, Estimating the Rényi entropy of several exponential populations, Brazilian Journal of Probability and Statistics, (2013).

[34] R. Koenker and I. Mizera, Quasi-concave density estimation, Annals of Statistics, 38 (2010) 2998-3027.

[35] S.F. Bagheri, M. Alizadeh, Baloui Jamkhaneh and S. Nadarajah, Evaluation and comparison of estimations in the generalized exponential-Poisson distribution, Journal of Statistical Computation and Simulation, 84(11) (2014) 2345-2360. DOI:10.1080/00949655.2013.793342.

[36] S.F. Bagheri, M. Alizadeh, S. Nadara jah and E. Deiri, Efficient estimation of the PDF and the CDF of the Weibull extension model, Communications in Statistics-Simulation and Computation, 45(6) (2016) 2191-2207.

[37] S. Nadarajah, The exponentiated Gumbel distribution with climate application, Environmetrics, 17 (2005) 13-23.

[38] T.A. Raja and A.H. Mir, On extension of some exponentiated distributions with application, International Journal of Contemporary Mathematical Sciences, 6 (2011) 393-400.

[39] W.Z. Lech and G. Maryna, The best measurand estimators of trapezoidal PDF, in Proceedings of the XIX Imeko World Congress, (2009) 2405-2410.

[40] Y. Fang, Asymptotic equivalence between cross-validations and Akaike Information Criteria in mixedeffects models, J. of Data Science, 9 (2011) 15-21. 